\title{
SOURCE ENHANCED CONDENSATION OF A SINGLE-COMPONENT VAPOR IN THE TRANSITION REGIME
}

\author{
A. A. Lushnikov, ${ }^{*}$ M. Kulmala, ${ }^{\dagger}$ H. Arstila ${ }^{\dagger}$ and E. L. Zapadinsky ${ }^{\ddagger}$ \\ * Karpov Institute of Physical Chemistry, 10, Vorontsovo Pole, 103064 Moscow K-64, Russia \\ ${ }^{\dagger}$ Department of Physics, Helsinki University, P.O. Box 9, FIN-00014, Helsinki, Finland \\ ${ }^{\ddagger}$ Institute of Chemical Kinetics and Combustion, SB, RAS 630090 Novosibirsk, Russia
}

\begin{abstract}
The growth of aerosol particles by homogeneous condensation in the presence of a spatially uniform source of a condensing monocomponent vapor is investigated. The kinetic model in which the condensation growth obeys the set of formal kinetic equations describing the process $(g)+(1) \rightarrow(g+1)$ (with $g$ being the number of vapor molecules in a particle) is used. The mass spectrum is expressed in terms of the function describing time evolution of the vapor concentration. In contrast to the case of free condensation (no external source), the mass spectrum is a smooth function of the particle mass with the frontal peak moving with time to the right along the mass axis. We have shown (Lushnikov and Kulmala, 1995, Phys. Rev. E 52, 1658-1668) that the particle number concentration either grows unlimitedly with increasing time or remains finite depending on how fast the condensation coefficients $\alpha_{g}$ describing the rates for monomer accretion grow with the particle mass $g$. For power dependencies $\alpha_{g} \propto g^{\lambda}$ the condensation regime switches at $\lambda=1 / 2$. The analogy of this phenomenon to the second-order phase transitions is notcd. The present numerical results illustrate the growth processes using condensation coefficients obtained from Fuchs and Sutugin (1970, J. Colloid Interface Sci. 27, 216-222). The analysis of transition regime shows that the particle mass spectrum can be expressed in terms of a universal function whereas the dependence on the Knudsen number enters in a rather trivial manner in the final expression for the mass spectrum. Copyright (C) 1996 Elsevier Science Ltd
\end{abstract}

\section{INTRODUCTION}

Although the investigation of formation and growth of aerosol particles by condensation has a long history, the first systematic analysis of the process was given by Nikolai Albertovich Fuchs in his popular book Evaporation and Droplet Growth in Gaseous Media (Fuchs, 1959). The main attention in this book was directed to the growth processes by diffusion of condensing substances onto the surfaces of single particles. In his later work written together with his most prominent student, Dr A. G. Sutugin (Fuchs and Sutugin, 1970), a very simple and still extremely effective empirical formula was proposed describing the vapor mass transport to a particle whose size is comparable or smaller than the mean free path of the vapor molecule in the carrier gas (transition regime). This Fuchs-Sutugin formula remains the most popular until now for two reasons: (i) it is simple and (ii) later studies of this process based on the analysis of the solution of the Boltzmann equation (Smirnov, 1987; Yalamov et al., 1981; Ivchenko, 1984; Ivchenko and Nikishin 1984; and many others) showed the Fuchs-Sutugin formula to hold within 10\% - the precision which is more than enough for all practical calculations nowadays.

Of course, Nikolai Albertovich Fuchs clearly understood the importance of the collective aspects of condensation process (the necessity to describe the process in terms of the particle-size distribution), and tried himself to approach this and other similar problems from this angle (Fuchs and Sutugin, 1968, 1970, 1971).

One of us (A.L.) worked with Nikolai Albertovich for eleven years watching his permanent and insistent attention to the problem of aerosol particle formation. Fuchs, e.g., initiated the activity of Dr A. G. Sutugin in this direction (Sutugin and Grimberg, 1976; Sutugin, 1971; Sutugin et al., 1981) and maintained considerable interest for the results of his work.

The interest for the condensational growth of aerosols has not faded. In fact, many new aspects appeared since the death of Nikolai Albertovich in the year 1982, among them:

- source enhanced condensation,

- condensation of vapor mixtures, especially binary condensation, 
- condensation-evaporation processes in clouds,

- condensational growth of irregular aggregates (fractals, in particular),

- condensational growth of "cosmosol" particles (i.e. without carrier gas), and

- condensational growth of nanometric particles

The order of the above list does not reflect their relative importance. In this paper we consider only the first item.

The situations when newly formed molecules of a condensing vapor appear during the course of the condensation process are not so rare (see e.g. Seinfeld, 1986; Warren and Seinfeld, 1984). Some examples are the condensation of vapors whose molecules are produced by chemical reactions [gaseous precursors, sulphuric acid in the atmosphere (Seinfeld, 1986; Van Dingenen and Raes, 1991; Kulmala et al., 1995)], aerosol production in an irradiated atmosphere (Subba-Ramu and Muraleedharan, 1986), as well as certain technological applications of the condensation processes (Tohno and Itoh, 1993; Ying, 1993).

Interest in the problem was renewed also in connection with non-aerosol applications of the source enhanced condensation process. A general statement of the problem is well described in the paper of Clement and Wood (1979) (see also the criterion of earlier works therein). The source enhanced polymerization was considered by Rabeony and Reiss (1988, 1989) and Bodalia et al. (1994), who analyzed the polymer chain growth by joining monomeric molecules. Some applications of source enhanced condensation theory to the growth of island films were reported by Blackman and Wilding (1991), who performed a scaling analysis of island mass spectra and found many scaling laws for time dependence of the moments of the mass distribution similar to those reported in this and a later paper (Blackman and Marshall, 1994). Lushnikov and Kulmala (1995) (referred hereafter to as LK) gave the general analysis of the time evolution of particle mass spectra in source enhanced condensing systems. The central results of this work are outlined here and used to demonstrate possible ways for the applications of the above theory to aerodisperse systems.

Below we study the following process: a spatially uniform source whose productivity $I(t)$ is a known function of time $t$ produces single molecules of the condensing substance. The molecules may be involved into the growth process in three ways:

- Two colliding vapor molecules form a stable dimer which then serves as a condensation nucleus continuing to grow by adding other monomeric vapor molecules (non-barrier nucleation).

- The vapor begins to nucleate. The nucleation process produces supercritical aerosol particles serving as condensation nuclei. Their production rate is proportional to the source intensity $I(t)$ and depends on the evaporation and condensation rates (the probability per unit time for a vapor molecule to escape from or condense on the particle).

- The vapor molecules condense on preexisting aerosol particles.

This paper deals only with the first mechanism of particle formation and growth. The collection of the condensing particles is assumed to be characterized by the particle mass spectrum $\left\{c_{q}(t)\right\}$, where $c_{q}(t)$ is the concentration of particles containing $g(g>1)$ monomers (in what follows, the vapor molecules will be referred to as monomers). The mass spectrum evolves owing to the chain of reactions $(g)+(1) \rightarrow(g+1)$ in each of which a monomer joins a $g$-mor at a given reaction rate $\alpha_{g}$. The external source pcrmanently adds monomers, thus leading to the growth of their concentration at the initial stage. The subsequent decrease of the monomer concentration is a consequence of the stable dimer formation and the accretion of the monomers onto the surface of formed $g$-mers.

Starting with the set of equations describing the kinetics of the condensation process in the presence of the external source LK have shown that the mass spectrum of the forming aerosol particles can be expressed in terms of the function describing the time dependence of the vapor concentration.

The main result of our earlier consideration was the recognition of the fact that the mass spectrum of condensing particles is a smooth curve with a frontal peak moving to the right 
along the mass axis. If the condensation rate (the probability per unit time to add a vapor molecule to a condensing particle) increases with the particle mass sufficiently fast, the particle number concentration remains finite despite a permanently acting source of fresh condensing matter. Otherwise, it grows unlimitedly with time (see LK). Similar result was derived by Blackman and Wilding (1991) from a scaling assumption on the time-mass dependence of the particle mass spectra. Here we show that this scaling assumption does not hold generally (except the case $\alpha_{g} \propto g$ ), although the power time dependencies of the distribution moments are well reproduced.

In the present paper we continue our recent investigations (LK) and study numerically how the aerosol concentration behaves once the condensation efficiencies obey the Fuchs-Sutugin formula.

The present paper is organized as follows. The next section gives a general statement of the problem and formulation of basic equations for the case of non-barrier nucleation. The third section shows how the mass spectrum can be expressed in terms of the function describing the time change of the vapor concentration which, in turn, is analyzed in Section 4 where a kind of phase transition in the behavior of condensing systems is recovered. It is shown that for power-like dependences of the condensation efficiencies on the particle mass, the particle number concentration is either finite (at power exponents exceeding 1/2) or grows without limit in time otherwise. The results of the numerical analysis using the Fuchs-Sutugin formula of the source enhanced condensation process are presented in Section 5. In the transition regime we assume that the condensation efficiency grows faster than $g^{1 / 2}$ at the initial stage and slower than $g^{1 / 2}$ at the final stage thereby passing the critical point somewhere in between. We discuss our numerical results in view of this transition in Section 6. Section 7 concludes the paper with several remarks concerning possible ways of extending the results of this paper and their possible applications.

\section{THE STATEMENT OF THE PROBLEM}

Let us consider a spatially uniform condensing system consisting of the carrier gas, condensing vapor and a spatially uniform source permanently producing vapor molecules with the rate $I(t)$ (the number of vapor molecules produced in a unit volume at a time). We assume:

- The molecules are able to produce aerosol particles along a scheme in which the molecules are added one by one:

$$
\begin{aligned}
(1)+(1) & \rightarrow(2), \\
(2)+(1) & \rightarrow(3), \\
\cdots & \rightarrow \cdots \\
(g)+(1) & \rightarrow(g+1) .
\end{aligned}
$$

It is essential that neither breakup processes nor collisions between growing particles are included. The dimers are thus assumed to be stable against breakup and can grow by accreting one monomer at a time.

- The rate $\alpha_{g}$ (condensation coefficients) of joining a monomer to $g$-mer is known as the function of the growing particle mass $g$ (the number of monomeric molecules comprising the particle). The Fuchs-Sutugin expression for $\alpha_{g}$ is adopted (Fuchs and Sutugin, 1970)

$$
\alpha_{g}=4 \pi D a_{1} f(g),
$$

where $D$ is the diffusion coefficient of the condensing molecules, $a_{1}=\left(3 v_{1} / 4 \pi\right)^{1 / 3}$ and $v_{1}$ is the volume per molecule in the growing particle. The $g$-dependence of the condensation coefficient $\alpha_{g}$ is given by the function $f(g)=g^{1 / 3} \phi(\zeta(g))$, where

$$
\phi(\zeta)=\frac{\zeta+1}{\frac{4}{3} \zeta^{2}+1.71 \zeta+1}
$$


and

$$
\zeta=\frac{\lambda_{\mathrm{m}}}{a_{1} g^{1 / 3}}
$$

Here $\lambda_{\mathrm{m}}$ is the effective mean free path of the vapor molecule. Hence, $\zeta$ is just the Knudsen number. Equation (1) is valid for all condensation regimes of interest. In the free molecular regime at $\zeta \gg 1$, equation (1) gives

and in the continuous regime $(\breve{b} \ll 1)$

$$
x_{g} \propto g^{2 / 3}
$$

$$
x_{g} \propto g^{1 / 3} .
$$

LK also analyzed other power dependences of $\alpha_{g}$ :

$$
\alpha_{q} \propto g^{\lambda} \text {. }
$$

- The source productivity is a known function of time $t$. Here we consider only sources independent of time.

Let $c_{1}(t), c_{2}(t), \ldots c_{g}(t)$ be the number concentrations of $1,2, \ldots g$-mers (mass spectrum). The set of equations describing their time evolution is

$$
\begin{gathered}
\frac{\mathrm{d} c_{1}}{\mathrm{~d} t}=I-c_{1} \sum_{g=1}^{\infty} \alpha_{g} c_{g}, \\
\frac{\mathrm{d} c_{2}}{\mathrm{~d} t}=\frac{1}{2} \alpha_{1} c_{1}^{2}-\alpha_{2} c_{1} c_{2}, \\
\vdots \\
\frac{\mathrm{d} c_{g}}{\mathrm{~d} t}=\alpha_{g-1} c_{1} c_{g-1}-\alpha_{g} c_{1} c_{g} .
\end{gathered}
$$

These equations have a quite transparent physical meaning. The change in the concentration of $g$-mers results from two processes: $(g-1)+(1) \rightarrow(g)$ and $(g)+(1) \rightarrow(g+1)$ (the first and the second terms of the r.h.s. of equation (9), respectively). While the first process adds $g$-mers into the aerosol, the second one leads to their losses which accounts for the opposite signs of these terms.

The first two equations of this set have a different structure than the others. Equation (8) contains $1 / 2$ before the first term on its right-hand side. This "one-half" factor reflects the identity of two colliding monomers. The r.h.s. of equation (7) describes the monomer production (the term $I(t))$ and the sink due to monomer capture by all forming particles and monomers themselves.

This set of equations should be supplemented with the initial conditions. We choose the latter in the form:

$$
c_{g}(0)=0,
$$

which corresponds to the "clean-of-aerosol" atmosphere at the initial moment (when the source begins to produce the precursor).

Let us write down some useful consequences of the above equations. On summing equations $(8),(9)$ over $g$ within the interval $[2, \infty)$ gives equation for the number concentration of aerosol particles:

$$
\frac{\mathrm{d} N}{\mathrm{~d} t}=\frac{1}{2} \alpha_{1} c_{1}^{2},
$$

with $N=\sum_{g=2}^{\infty} c_{g}$. The summation begins with $g=2$, for the monomeric molecules are attributed to the gaseous phase.

The second consequence reflects the conservation of the total mass concentration $M(t)=\sum_{g=2}^{*} c_{g}(t)$. Multiplying both sides of the set (9) by $g$ and summing again over all $g \geqslant 2$ yield $\dot{M}-I-\dot{c}_{1}$ or

$$
M=I t-c_{1} .
$$


The replacement of variables and unknown functions

$$
c_{g} \rightarrow \sqrt{\frac{I}{\alpha_{1}}} c_{g}\left(t \sqrt{I \alpha_{1}}\right)
$$

will sometimes allow us to set $I=1$ and $\alpha_{1}=1$.

\section{DERIVATION OF A MASS SPECTRUM}

The strategy for solving the infinite set of coupled nonlinear differential equations (7)-(9) includes the following five steps (see LK):

(1) Equations (8) and (9) are treated independently of equation (7), the concentration $c_{1}$ being considered as a given function of time.

(2) The new variable

$$
\tau=\int_{0}^{t} c_{1}\left(t^{\prime}\right) \mathrm{d} t^{\prime}
$$

is introduced instead of $t$. This step casts equations (8) and (9) into infinite set of uncoupled linear ordinary differential equations (Goodrich, 1964).

(3) We solve this set and express the spectrum in terms of $c_{1}(\tau)$.

(4) The spectrum thus found is used to close the equation for $c_{1}(\tau)$.

(5) The way back from $\tau$ to $t$ does not create problems once $c_{1}(\tau)$ is known:

$$
t=\int_{0}^{\tau} \frac{\mathrm{d} \tau}{c_{1}(\tau)}
$$

Let us start our way along this route. The first step has been done (see equation (14)). Now equation (8) takes the form

$$
\frac{\mathrm{d} c_{2}}{\mathrm{~d} \tau}=\frac{1}{2} \alpha_{1} c_{1}-\alpha_{2} c_{2}
$$

This equation contains the non-uniformity $c_{1}(\tau)$ which at this stage is considered as a known function of the new variable $\tau$. Other equations of this set are homogeneous:

$$
\frac{\mathrm{d} c_{g}}{\mathrm{~d} \tau}=\alpha_{g-1} c_{g-1}-\alpha_{g} c_{g}
$$

Equations (16) and (17) can be solved by the Green function method giving

$$
c_{g}(\tau)=\int_{0}^{\tau} \mathscr{G}_{g}\left(\tau-\tau^{\prime}\right) c_{1}\left(r^{\prime}\right) \mathrm{d} \tau^{\prime}
$$

where $\mathscr{G}_{g}(\tau)$ is the Green function of the set (8) and (9), i.e. its solution with $c_{1}$ replaced by Dirac's delta-function $\delta(\tau)$. The asymptotic of LK showed that at sufficiently large $g$ and $\tau$ the Green function has the Gaussian form:

$$
\mathscr{G}_{g}(\tau) \approx \sqrt{1 / 2 \pi \Gamma_{g}^{2}} \exp \left[-\left(\tau-\tau_{g}\right)^{2} / 2 \Gamma_{g}^{2}\right],
$$

where the position of the Gaussian peak is

$$
\tau_{g}=\sum_{s=2}^{g} x_{s}^{-1}
$$

and its width is expressed in terms of the condensation efficiencies as follows:

$$
\Gamma_{g}=\sqrt{\sum_{s=2}^{g} \alpha_{s}^{-2}}
$$


The following essential moments should be marked:

(a) The sum on the r.h.s. of equation (21) converges at large $g$ for $\lambda>1 / 2$ and diverges otherwise, giving the first indication that $\lambda=1 / 2$ represents a "singular" point.

(b) The ratio of the width to the peak position always decreases with growing $g$, allowing the replacement of the Gaussian by a delta function when it multiplies smooth functions of $\tau$ or $g$.

Equations (18) and (19) formulate the final result of this section:

$$
c_{g}(\tau)=\frac{\alpha_{1}}{2 \alpha_{g} \sqrt{2 \pi \Gamma_{g}^{2}}} \int_{0}^{\tau} \exp \left[-\frac{\left(\tau-\tau^{\prime}-\tau_{g}\right)^{2}}{2 \Gamma_{g}^{2}}\right] c_{1}\left(\tau^{\prime}\right) \mathrm{d} \tau^{\prime} .
$$

Now everything is known except the monomer concentration, for which the closed equation was derived in LK:

$$
c_{1} \frac{\mathrm{d} c_{1}}{\mathrm{~d} \tau}=I-\alpha_{1} c_{1}^{2}-\frac{1}{2} \alpha_{1} c_{1} \int_{0}^{\tau} \alpha\left(\tau-\tau^{\prime}\right) c_{1}\left(\tau^{\prime}\right) \mathrm{d} \tau^{\prime} .
$$

Here the function $\alpha(\tau)=\alpha_{g(\tau)}$ is introduced with the dependence $g(\tau)$ being defined from the equality:

$$
\tau=\int_{2}^{g(\tau)} \frac{\mathrm{d} s}{\alpha_{s}}
$$

The further steps include numerical or analytical solution of equations (23), (24) and performing the integration in equation (22).

\section{THE PHASE TRANSITION}

In order to understand the physical picture, we give a simplified analytical treatment of the phenomenon. First, we approximate the sum on the r.h.s. of equation (7) as follows:

$$
\sum_{g=1}^{\infty} \alpha_{g} c_{g} \approx \alpha_{g(t)}\left(N(t)+c_{1}(t)\right)
$$

where the average particle mass $g(t)$ is defined as the ratio of the mass to number concentrations:

$$
g(t)=\frac{t}{N(t)+c_{1}(t)} .
$$

To derive equation (26) we used equation (12) and set $I=1$ in agreement with equation (13). The term $c_{1}(t)$ in the denominator of equation (26) is negligible compared to $N(t)$ at sufficiently large $t$. Now equations (7) and (11) give the closed set of first-order differential equations:

$$
\begin{gathered}
\frac{\mathrm{d} c_{1}}{\mathrm{~d} t}=1-\alpha(t / N) c_{1}\left(N+c_{1}\right), \\
\frac{\mathrm{d} N}{\mathrm{~d} t}=\frac{1}{2} c_{1}^{2} .
\end{gathered}
$$

Next, we return to $\alpha_{g}=g^{\lambda}$ and neglect the term $\mathrm{d} c_{1} / \mathrm{d} t$ on the 1.h.s. of equation (27) and $c_{1}$ in the brackets on its r.h.s. The latter approximations obviously hold at $t \gg 1$ meaning simply that all monomers are consumed by the particles formed at earlier stages of the condensation process. Now equation (27) allows $c_{1}$ to be expressed in terms of $N$ and $t$ to close equation (28):

$$
\frac{\mathrm{d} N}{\mathrm{~d} t}=\frac{1}{2} t^{-2 \lambda} N^{-2(1-\lambda)}
$$

Equation (29) is readily solved giving the asymptote shown in Table 1. 
Table 1. Asymptotic time behavior of monomer and particle number concentrations $(t, \tau \gg 1)$

\begin{tabular}{lllll}
\hline & $0 \leqslant \lambda<1 / 2$ & $\lambda=1 / 2$ & $1 / 2<\lambda<1$ & $\lambda=1$ \\
\hline$c_{1}(t)$ & $t^{-1 /(3-2 \lambda)}$ & $1 / \sqrt{t \sqrt{\ln t}}$ & $t^{-\lambda}$ & $t^{-1}$ \\
$N(t)$ & $t^{(1-2 \lambda) /(3-2 \lambda)}$ & $\sqrt{\ln t}$ & $N_{\infty}(\lambda)$ & $(\pi \sqrt{\pi}) / 4$ \\
\hline
\end{tabular}

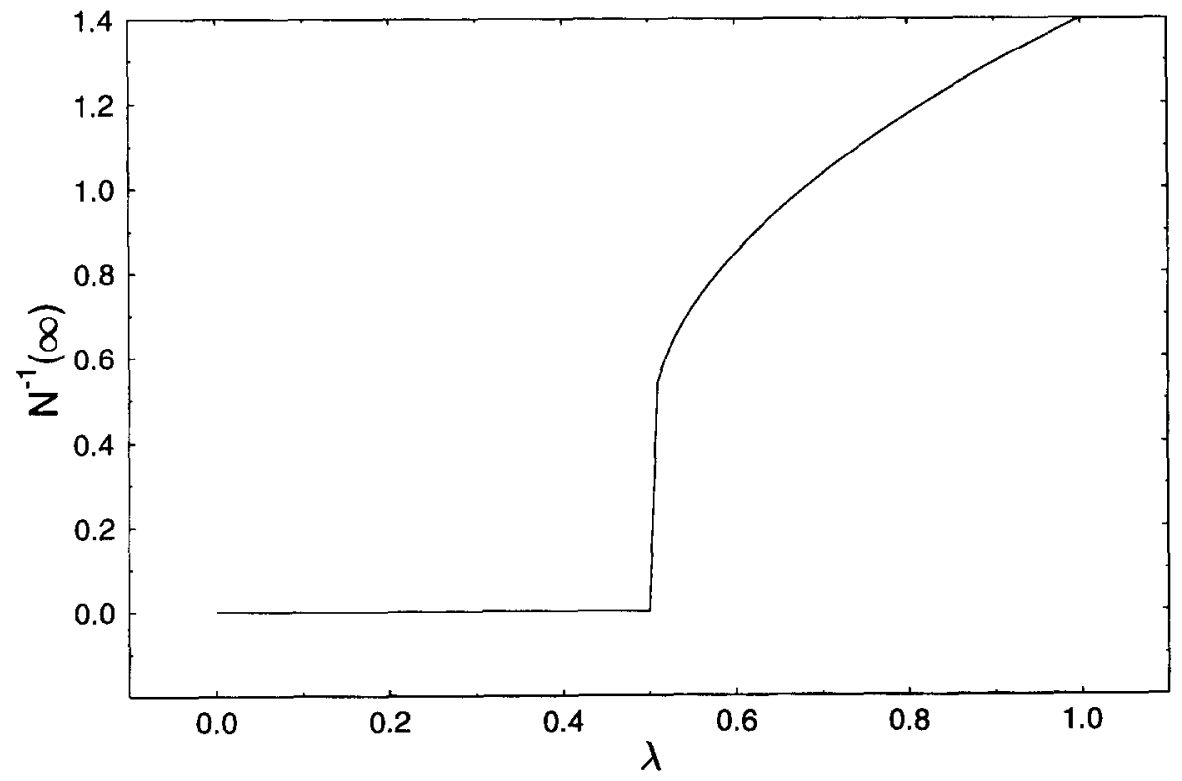

Fig. 1. Phase transition in the source enhanced condensing systems. The dependence of the "order parameter" $N^{-1}(t=\infty)$ on the power exponent $\lambda$. At $\lambda>1 / 2$ the condensation process ceases producing new particles at large times. All vapor produced by the source is spent by the growing particles formed at earlier stages of the condensation process.

Table 1 shows that the exponents in the power dependencies are continuous functions having jumps in their first derivative at $\lambda=1 / 2$. At $\lambda>1 / 2$, the number concentration remains finite at large $t$. The analogy between such unusual behavior and the second-order phase transition is clearly seen. $N_{\infty}(\lambda)$ stands for a finite, $\lambda$ dependent limit.

If we consider $p(\lambda)=N^{-1}(\infty)$ as the "order parameter" the analogy with phase transition will be even more apparent: $p(\lambda)=0$ at $\lambda \leqslant 1 / 2$ and $p(\lambda)>0$ otherwise (see Fig. 1).

Of course, equations (27) and (28) may be used for the approximate determination of $c_{1}(\tau)$ and to restore the mass spectrum along the route outlined in the previous section.

The whole situation may be summarized as follows: the source enhanced condensational growth of aerosol particles proceeds qualitatively differently with $\lambda<1 / 2$ and $\lambda>1 / 2$ in equation (6). When the condensation efficiency increase with particle mass is sufficiently slow (slower than $\alpha_{g} \propto \sqrt{g}$ ), the vapor consumption by formed aerosol particles never reaches the level sufficient for complete suppressing the new particle formation. The particle number concentration increases unlimitedly at $\tau \rightarrow \infty$ (and thus $t \rightarrow \infty$ ). At faster increase of $\alpha_{g}$ with $g$, the growing aerosol particles consume almost all vapor produced by the source preventing thereby the formation of the new particles. The particle number concentration remains finite at far stages of the condensation process.

Similar result was reported by Blackman and Wilding (1991), who started with the assumption on the scaling behavior of the particle mass spectrum at large $t$ :

$$
c_{g}(t)=g^{-\theta} f\left(g / t^{z}\right),
$$


where the power exponents $\theta$ and $z$ were determined by Blackman and Wilding (1991). The scaling assumption is seen to be in contradiction with our asymptotically exact result (equation (22)) giving, nevertheless, correct values of respective power exponents as well as our approximate approach.

\section{TRANSITION REGIME}

In this section we apply the above theory to the transition regime. The first step along this route is the introduction of properly arranged condensation efficiency instead of that given by the Fuchs-Sutugin formula (equation (2)):

$$
\alpha_{g}=\alpha_{1} g^{2 / 3} \frac{1+\xi g^{1 / 3}}{1+1.283 \xi g^{1 / 3}+0.75 \xi^{2} g^{2 / 3}},
$$

where the dimension carrier is $\alpha=3 \pi D a_{1}^{2} / \lambda_{m}$ and the parameter $\xi=a_{1} / \lambda_{m}$ is always small $\left(\propto 10^{-3}\right.$ at normal conditions) and ignored everywhere except those cases when this parameter appears in the combination $g^{1 / 3} \xi$.

Next, we introduce the dimensionless time $t$ measured in units of $\left(I \alpha_{1}\right)^{-1 / 2}$, the condensation efficiency $\alpha_{g}$ measured in units of $\alpha_{1}$ and concentrations $c_{g}(t)$ measured in units of $\sqrt{I / \alpha_{1}}$ in accordance with equation (13).

Next all the functions entering equations (22) and (23) are calculated.

Replacing the sum in equation (20) by an integral and subsequent elementary

$$
\tau+3.780=1.6 g^{1 / 3}+1.125 \xi g^{2 / 3}+\frac{1.4}{\xi} \ln \left(1+\xi g^{1 / 3}\right) .
$$

This equation defines the dependence $g(\tau)$ (see also equation (24)).

The width $\Gamma_{g}$ (equation (21)) becomes:

$$
\Gamma_{g}^{2}=2.381-3 g^{-1 / 3}+\xi\left[1.689 \xi g^{1 / 3}+2.4 \ln \left(\xi g^{1 / 3}\right)-1.698 \ln \xi\right] .
$$

Only terms growing with $g$ and $\xi$ are retained in the brackets. It is of interest to follow the structure of this expression. It contains a constant term and terms growing with $g$ which, however, are of importance only at very large $\xi g^{1 / 3}$ when the combination $\xi^{2} g^{1 / 3}$ becomes of the order of unity. This fact allows one to ignore all terms in the brackets except the first one and to rewrite the above equation in a simpler form:

$$
\Gamma_{g}^{2}=2.381-3 g^{-1 / 3}+1.689 \xi^{2} g^{1 / 3} .
$$

One sees that at $\xi^{2} g^{1 / 3} \ll 1$, the width is independent of $g$.

In order to reach the continuous regime, it is necessary to travel a long way along the $g$-axis: corresponding values of $g$ have the order of $10^{12}$. Corresponding interval of $\tau$ also spans four orders of magnitude. These two facts prevent a direct computer solution of this problem and necessitate an approximate semianalytical approach based on the following simple idea: to perform direct numerical integration of equation (23) and then use a version of the asymptotic approach of LK. Because the function $\alpha_{g}$ is not a power function of $g$, the straightforward application of the LK theory is impossible. Nevertheless, some simplifications are still available.

The numerical integration of equation (23) shows that the function $c(\tau)$ has a sharp maximum at $\tau \propto 1$ which allows one to replace the very smooth function $\alpha\left(\tau-\tau^{\prime}\right)$ in the integrand of equation (23) with $\alpha(\tau)$ and solve the following set of two ordinary differential equations:

$$
\begin{gathered}
c_{1} \frac{\mathrm{d} c_{1}}{\mathrm{~d} \tau}=1-c_{1}^{2}-c_{1} \alpha(\tau) N, \\
\frac{\mathrm{d} N}{\mathrm{~d} \tau}=\frac{1}{2} c_{1} .
\end{gathered}
$$


Next, at large values of $t$ the l.h.s. of equation (35) and the second term on the r.h.s. of this equation may be ignored (see LK) allowing thereby the solution of equations (35) and (36) in the form

$$
\begin{gathered}
N(\tau)=\sqrt{N^{2}\left(\tau_{0}\right)+\int_{\tau_{0}}^{\tau} \mathrm{d} \tau^{\prime} / \alpha\left(\tau^{\prime}\right)}, \\
c_{1}(\tau)=\frac{1}{\alpha(\tau) N(\tau)} .
\end{gathered}
$$

The integral on the r.h.s. of equation (37) is also readily expressed in terms of $\Gamma_{g}$ :

$$
\int_{\tau_{0}}^{\tau} \frac{\mathrm{d} \tau^{\prime}}{\alpha\left(\tau^{\prime}\right)}=\int_{g\left(\tau_{0}\right)}^{g(\tau)} \frac{\mathrm{d} s}{\alpha_{s}^{2}}=\Gamma^{2}(g(\tau))-\Gamma^{2}\left(g\left(\tau_{0}\right)\right) .
$$

In deriving the above result equations (21), (24) and (33) were used. Equation (32) defines the dependence $g(\tau)$.

The following iterative method was used to solve $c_{1}(\tau)$ :

Equations (35) and (36) can be written in the form

$$
\begin{gathered}
\frac{\mathrm{d} f}{\mathrm{~d} \tau}=2(1-f-\sqrt{f} \alpha N), \\
\frac{\mathrm{d} N}{\mathrm{~d} \tau}=0.5 \sqrt{f},
\end{gathered}
$$

where $f=c_{1}^{2}$. The set of differential equations (40) and (41) was solved numerically for $\tau \leqslant 1000$ with initial conditions $f(0)=c_{1}(0)=0$ and $N(0)=0$. The resulting $c_{1}(\tau)$ is used in equation (23) under the integral sign. Equation (23) is also written in terms of $f=c_{1}^{2}$ and it is solved with the initial conditions mentioned above to get a new approximation for $c_{1}(\tau)$, which is again used under the integral sign to get another approximation for $c_{1}(\tau)$. The convergence of this iterative method is demonstrated in Fig. 2. Four steps are enough for successive approximations to be practically the same.

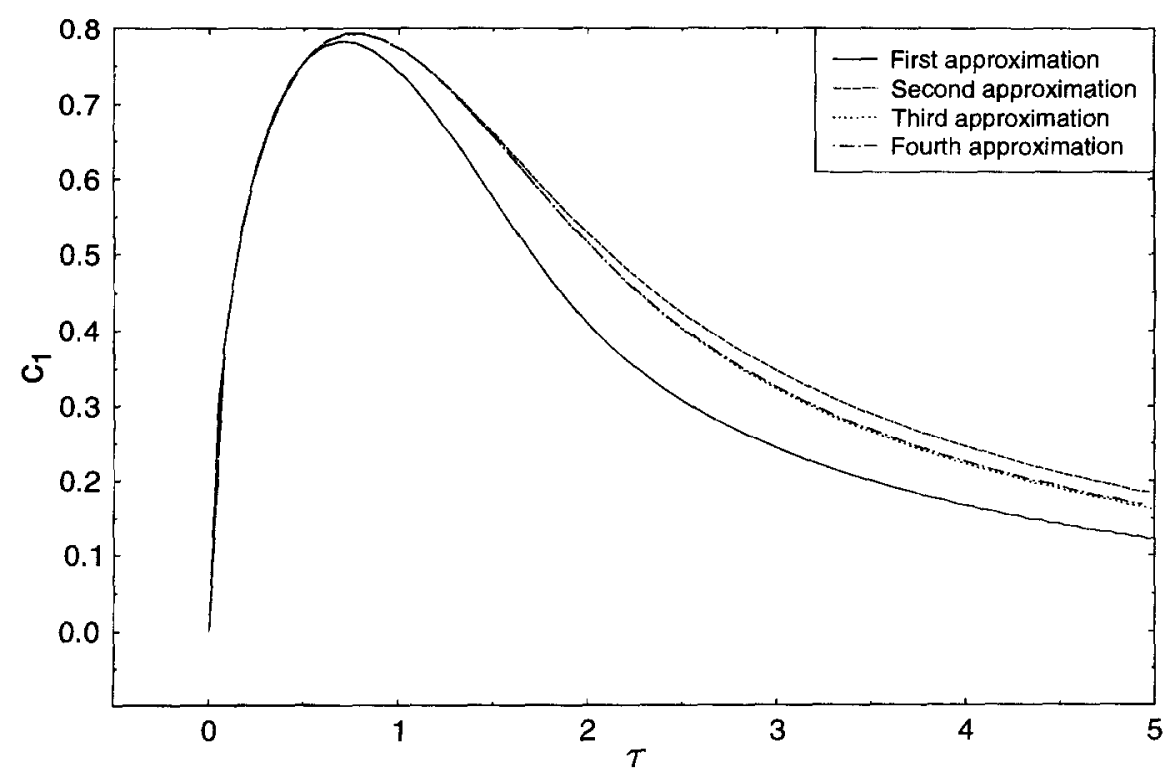

Fig. 2. The results of successive iterative steps for solving equation (23). First approximation is given by the solution of (35) and (36). Approximation for $c_{1}(\tau)$ is used under the integral sign in equation (23), which is solved to get the next approximation. Three steps are enough to get indistinguishable curves. 
In calculating the mass spectra at large $\tau$, we cut the upper limit in the integral in equation (22) by putting it equal to $\tau_{0}$. The reason for this step is the smallness of $c_{1}(\tau)$ at larger $\tau$.

The dependence on $\xi$ in $\Gamma_{g}$ may be neglected at $g<10^{12}$. Equation (34) justifies this approximation: the product $\xi^{2} g^{1 / 3}$ is of the order of $10^{-2}$.

This allows the size spectrum to be expressed in terms of a single universal function almost independent of $\xi$ :

$$
c_{g}(\tau)=\frac{1}{2 \alpha_{g} \sqrt{2 \pi \Gamma_{g}^{2}}} F_{g}(\tau)
$$

where

$$
F_{g}(\tau)=\int_{0}^{\tau_{0}} \exp \left[-\frac{\left(\tau-\tau_{g}-\tau^{\prime}\right)^{2}}{2 \Gamma_{g}^{2}}\right] c_{1}\left(\tau^{\prime}\right) \mathrm{d} \tau^{\prime}
$$

The function $F_{g}(\tau)$ is claimed to be almost independent of $\xi$ although the function $c_{1}(\tau)$ enters the integrand on the r.h.s. of equation (43), because at $\tau<\tau_{0}$, the condensation in the free molecular regime defines the time behavior of the monomer concentration, or, shorter, the parameter $\xi$ may be neglected at such $\tau$ in calculating the function $c_{1}(\tau)$. The dependence of the condensation process on the Knudsen number enters only via the condensation efficiencies $\alpha_{g}$ and the time dependence of the maximum position $\tau_{g}$. In practice, $\xi$ changes only slightly when varying physical conditions. Our numerical analysis shows (see Section 6) that $F_{g}$ is numerically almost independent of $\xi$.

Now rather simple numerical calculations allow the full analysis to be completed.

\section{RESULTS AND DISCUSSION}

In this paper we outlined the general and asymptotically exact approach developed in LK and demonstrated its application to the realistic situation of the source enhanced condensational formation and growth of aerosol particles in the transition regime. The Fuchs-Sutugin semiempirical expression for the condensation efficiencies (equation (2)) combined with LK approach allowed the whole problem to be reduced to the numerical solution of the ordinary integrodifferential equation $(23)$ for $c_{1}(\tau)$ and some rather elementary analytical or numerical integrations. Introducing dimensionless mass spectrum and

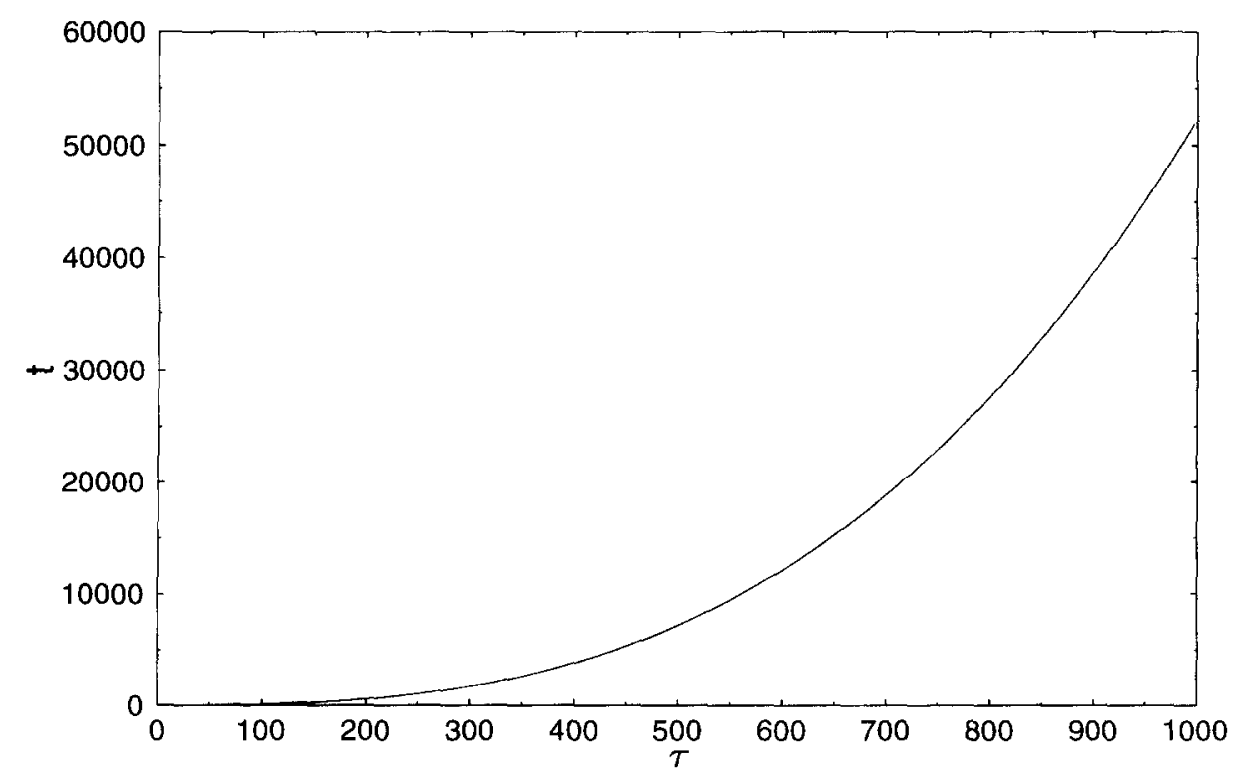

Fig. 3. Source enhanced condensation of aerosols in the transition regime. The dependence of variable $\tau$ on real dimensionless time. 
time (equation (13)) gives the results expressed in terms of the universal (independent of the source productivity $I$ and the dimension carrier $\alpha_{1}$ and the parameter $\xi$ ) function $F_{g}(\tau)$. Figure 3 shows the dependence of real dimensionless time $t$ on $\tau$. One clearly sees why the use of the variable $\tau$ is much more favorable than $t$ : very large $t$ corresponds to much smaller $\tau$ which substantially reduces computing times. The condensation coefficient $\alpha_{g}$ (equation (31)) and the maximum position $\tau_{g}$ are plotted in Fig. $4 \mathrm{a}$ and $\mathrm{b}$ versus the particle mass $g$.

The $\tau$-dependence of the monomeric and total number concentrations are displayed in Fig. 5. One clearly sees that the maximum is very narrow, which justifies the approximations described in Section 5. The function $N(\tau)$ is given by solution of partial differential equations (35) and (36) when $\tau \leqslant 1000$, and by equation (35) when $\tau>\tau_{0}=1000 . N(\tau)$ levels to a constant value of about 1.45 when $\tau=100$, growing very slowly after that.
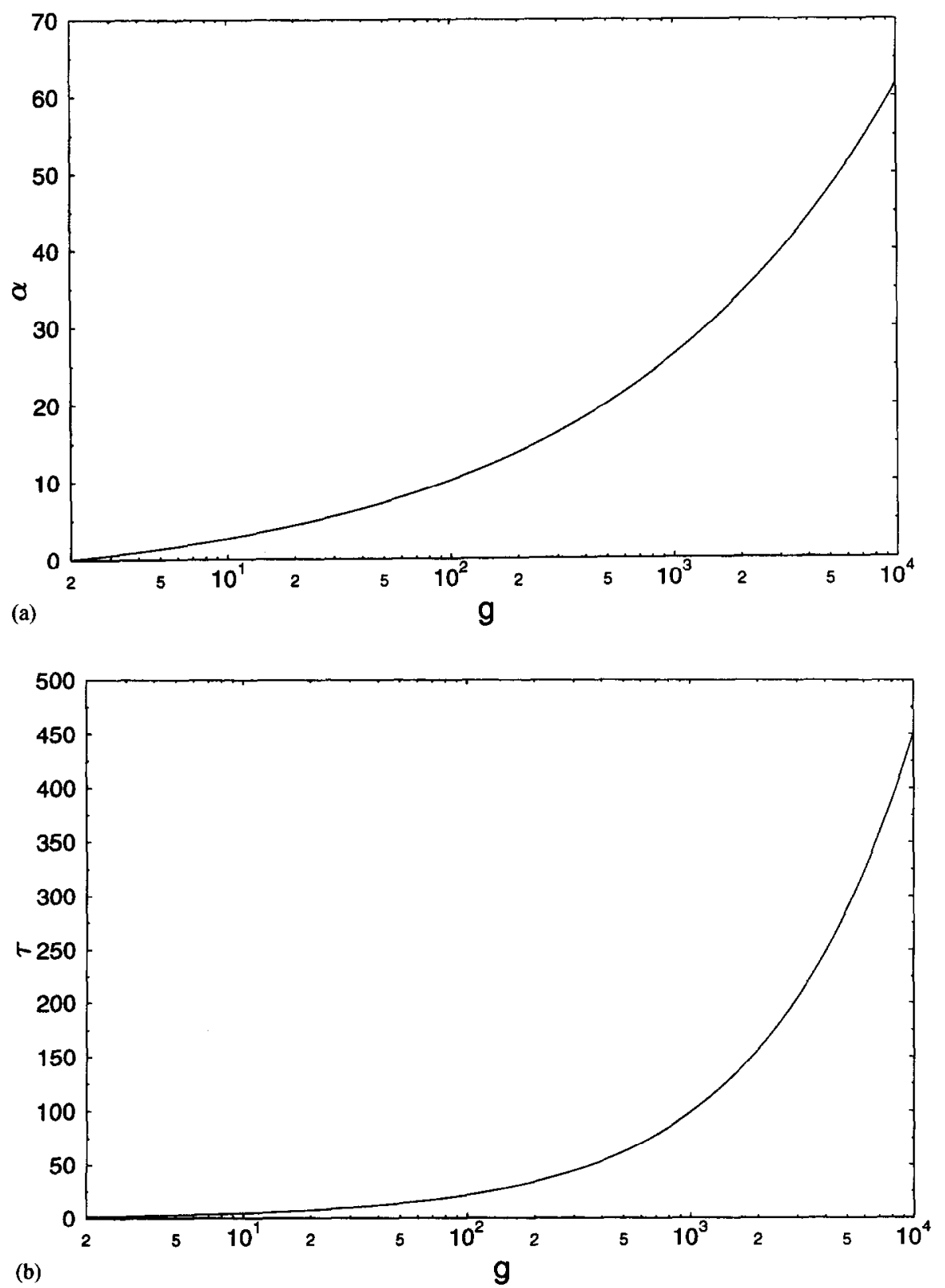

Fig. 4. Source enhanced condensation of aerosols in the transition regime. Condensation efficiency $\alpha_{g}(a)$ and the maximum position $\tau_{g}$ (b) are plotted versus $g$. 


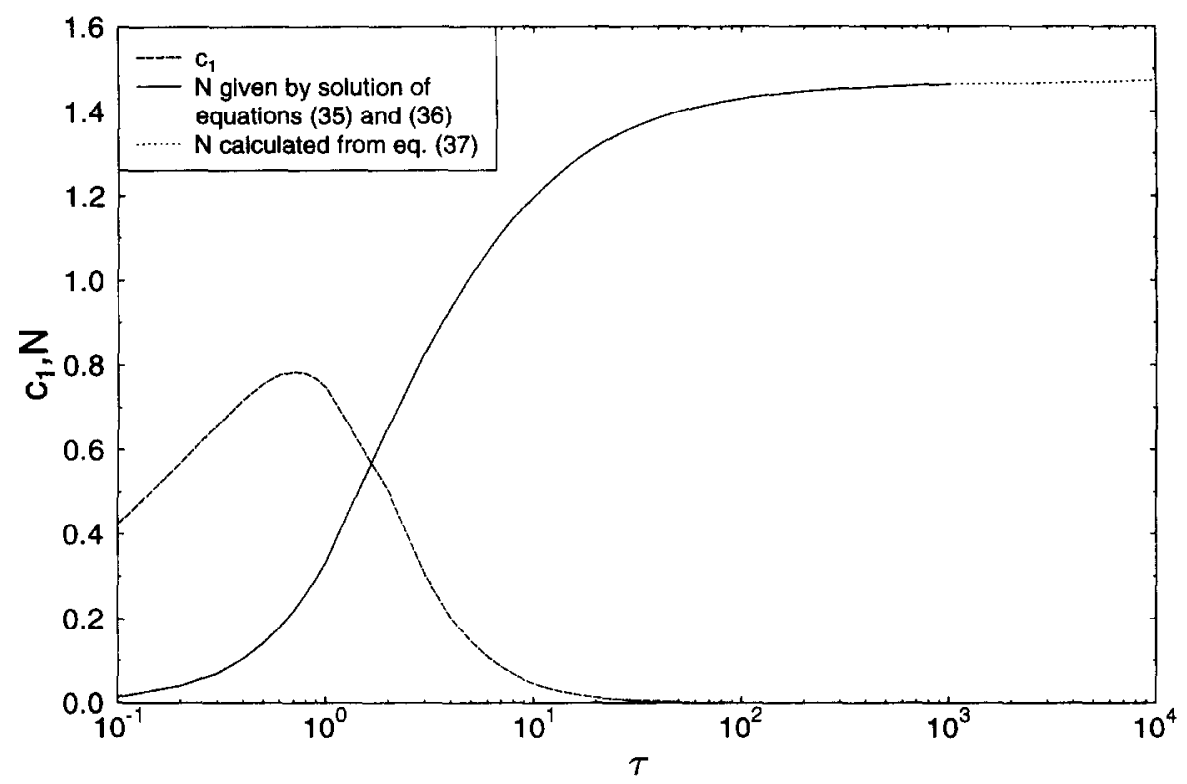

Fig. 5. Source enhanced condensation of aerosols in the transition regime. The monomer and particle number concentrations are shown as functions $\tau$.

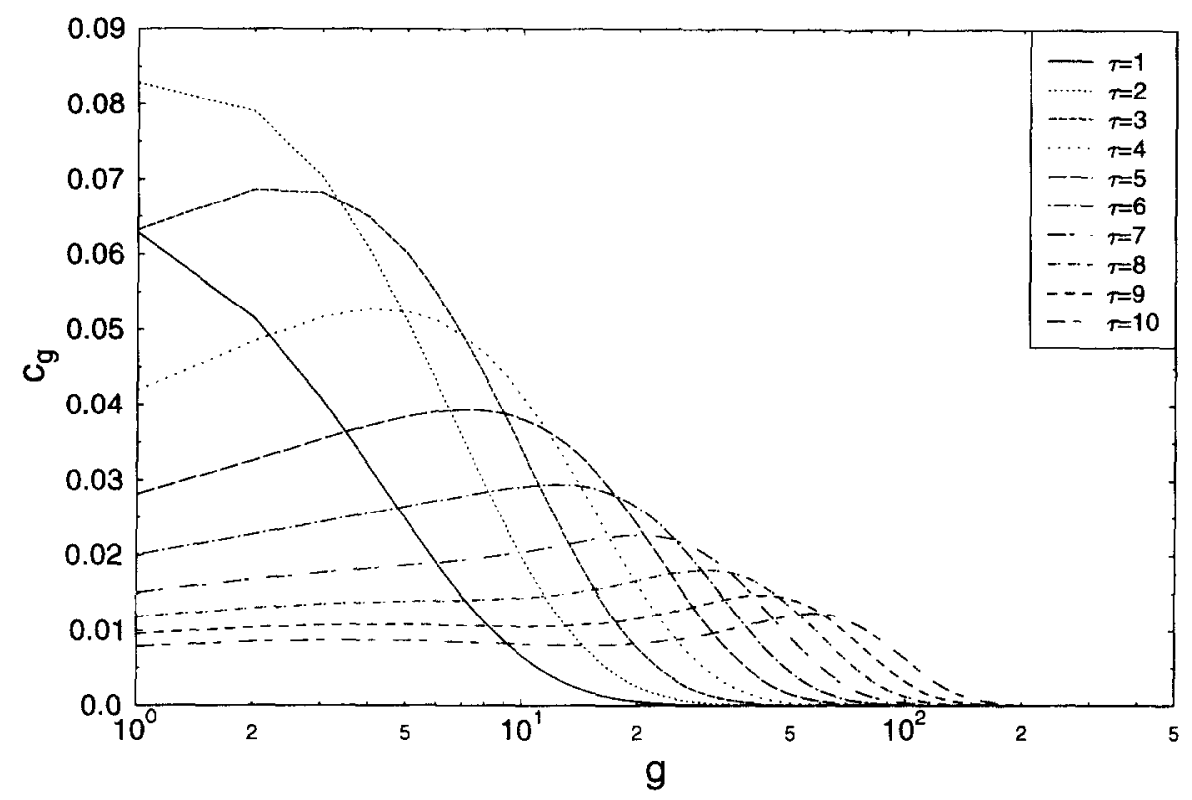

Fig. 6. Source enhanced condensation of aerosols in the transition regime. The time evolution of the size spectrum $c_{g}(\tau)$.

Figure 6 shows the time evolution of the size spectrum when $\tau$ is 10 or less. Figures $7 \mathrm{a}$ and b show the universal function $F_{g}(\tau)$ describing the particle mass spectrum with small and large values of $\tau$. It is important to stress once again that neither the properties of the carrier gas nor physicochemical constants of condensing substance enter this function.

Calculations with $\xi \cdot 10^{3}=1,2,3,4$ showed that all the results are not very sensitive to the value of $\xi$. In the figures presented here the curves corresponding to different values of $\xi$ would be indistinguishable. The results presented are calculated with $\xi \cdot 10^{3}=4$.

Perhaps the most remarkable feature of the Fuchs-Sutugin condensation efficiency is a smooth transition through the critical point $\lambda=\lambda_{\mathrm{c}}=1 / 2$. It is interesting to follow 

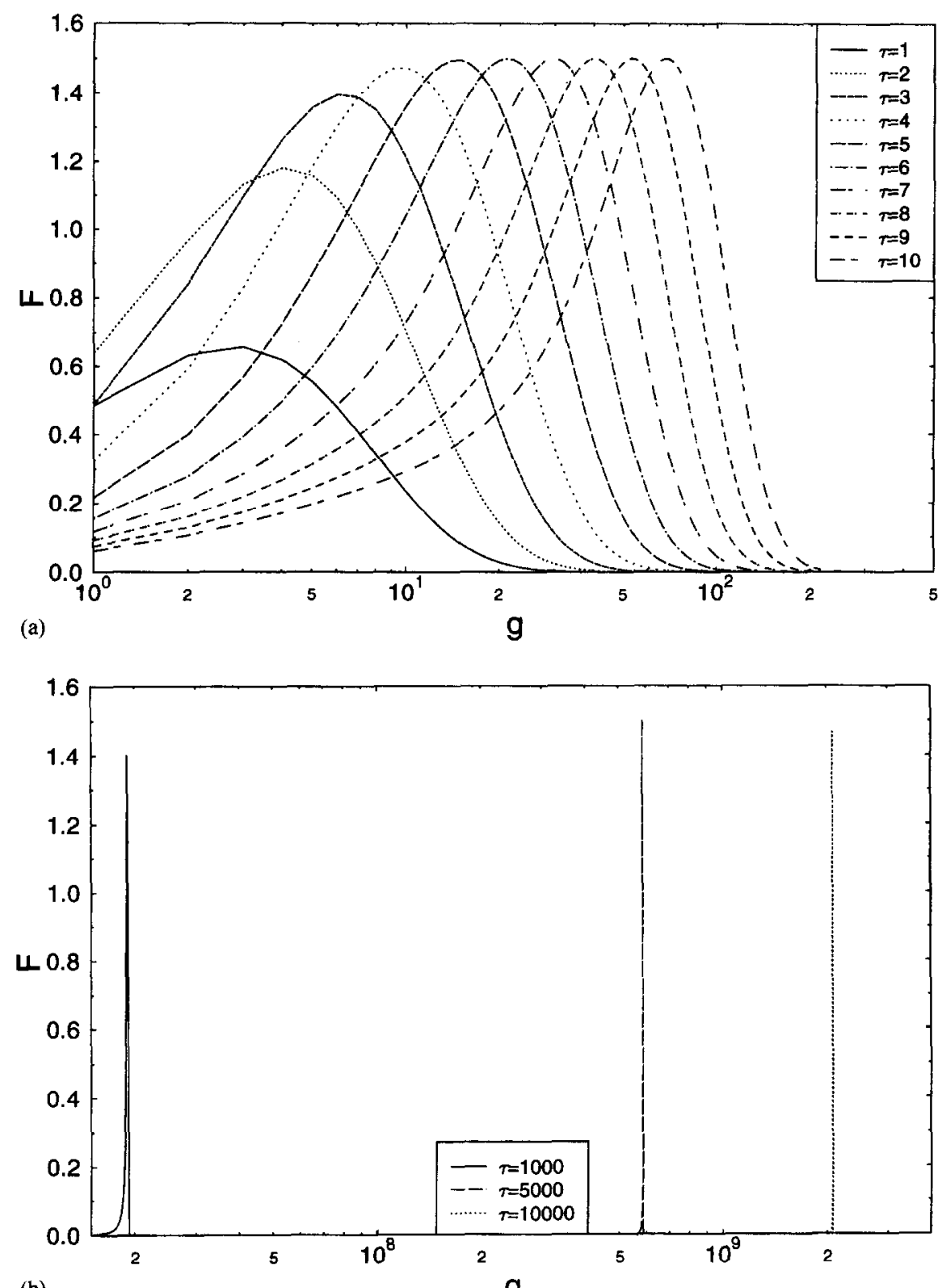

(b)

Fig. 7. Source enhanced condensation of aerosols in the transition regime. The universal function $F_{g}(\tau)$ describing the particle mass spectrum.

whether some critical phenomena come up in passing from free molecular to continuous regime of the particle growth. (from $\lambda=2 / 3$ or $\lambda=1 / 3$ ). Our analysis clearly demonstrates that neither jumps nor cusps are observed in the time behavior of the particle mass spectum and its moments. The only manifestation of the transition is the time dependence of particle number concentration which almost saturates at the free molecular stage (small $g$ at which $\alpha_{g} \propto g^{2 / 3}$ ) but continued growing due to the transition to diffusion controlled regime.

\section{CONCLUDING REMARKS}

Starting with the formal kinetic scheme we considered in detail the particle formation by condensation in presence of a spatially uniform and constant external source producing the molecules of a condensing vapor. We have shown that for realistic (in the present paper) and 
model condensation rates (typically growing with the particle mass as its power; see LK) the mass spectra of forming particles are described by rather smooth functions linearly linked with the time dependence of the monomer concentration. This result reduces the rather complicated problem of solving an infinite set of nonlinear differential equations to the integration of a single ordinary integrodifferential equation.

The most important physical consequence of the above theory is the existence of two types of mass spectra. When the condensation rate $\alpha_{g}$ grows slowly with the particle mass (slower than $\sqrt{g}$ ), the mass spectrum has a non-integrable peak at its front which moves to the right along the mass axis. The number concentration of the aerosol particles reveals unlimited growth with time.

For stronger dependencies of $\alpha_{g}$ on $g$ the maximum becomes integrable. The most remarkable fact in this case is finiteness of the degree of gas-to-particle conversion: the number concentration of the aerosol particle formed by the condensation process remains finite although the source continuously produces new condensing matter.

In both case above the mass spectrum has the frontal maximum (integrable or nonintegrable) and a long power tail. The monomer concentration decreases with time despite the presence of the source. This is not a surprising fact, for the vapor consumption grows with increasing particle size and the source has no time to produce enough vapor molecules to support the monomer concentration at a given level.

In the present paper we demonstrated the effectiveness of the LK approach in considering the aerosol particles growth in the transition regime. We showed that the whole theoretical consideration is reduced to several more or less elementary analytical or numerical steps and allows one to describe the final spectrum in terms of the universal function $F(x)$. Although the Fuchs-Sutugin formula describes a transition of the condensation regime through the critical point $\lambda=\lambda_{\mathrm{c}}=1 / 2$, no specific manifestations of the phase transition are observed. The particle number concentration almost saturates at times corresponding to the transition regime.

This work was primarily motivated by a necessity to create a sufficiently simple approach to attack much more complicated and more realistic situations including binary mixtures, a nucleation stage, or the presence of condensation nuclei.

The numerical analysis was carried out using MATLAB and NAG-Library Fortran routines D02EAF and D01GAF.

\section{REFERENCES}

Blackman, J. A. and Marshall, A. (1994) Coagulation and fragmentation in cluster-monomer reaction models. J. Phys. A: Math. Gen. 27, 724-740.

Blackman, J. A. and Wilding, A. (1991) Scaling theory of island growth in thin films. Europhys. Lett. 16, 115-120.

Bodalia, R., Manzanares, J., Reiss, H. and Duran, R. (1994) Kinetics of 2-pentadecylaniline polymerization in monolayers: relationship between experimental data and a new theoretical model. Macromolecules 27, 2002-2007.

Clement, C. F. and Wood, M. H. (1979) Equation for the growth of a distribution of small physical objects. Proc. Roy. Soc. Lond. A368, 521-546.

Fuchs, N. A. (1959) Evaporation and Droplet Growth in Gaseous Media. Pergamon Press, London.

Fuchs N. A. and Sutugin A. G. (1968) Formation of condensation aerosols at high vapor supersaturation. $J$. Colloid Interface Sci. 27, 216-222.

Fuchs, N. A. and Sutugin, A. G. (1970) Formation of condensation aerosols at rapidly changing environmental conditions. 1. Theory. J. Aerosol Sci. 1, 1287-1294.

Fuchs, N. A. and Sutugin, A. G. (1971) Noncoagulated highly dispersed aerosols. J. Aerosol Sci., 2, $371-379$.

Goodrich, F. C. (1964) Proc. Roy. Soc. Lond. A277, 155-166.

Ivchenko, I. N. (1984) A study of condensational growth and evaporation of water droplets in air. Dokl. Akad. Nauk SSSR 274, 572-575.

Ivchenko, I. N. and Nikishin, V. A. (1984) An extension of theory of evaporation of spherical droplets in diffusion regime. Dokl. Akad. Nauk SSSR 277, 1111-1116.

Kulmala, M., Kerminen, V.-M. and Laaksonen A. (1995) Atmos. Envir. 29, 377-382.

Lushnikov, A. A. and Kulmala, M. (1995) Source-enhanced condensation in monocomponent disperse systems. Phys. Rev. E, 52, 1658-1668.

Rabeony, H. and Reiss, H. (1988) Nonterminated chain polymerization in a convective gas. Macromolecules 21, 912-918.

Rabeony, H. and Reiss, H. (1989) Exact solution of boundary value problems involving linear processes of growth and transport in addition polymerization. J. Chem. Phys. 91, 1875-1881. 
Seinfeld, J. H. (1986) Atmospheric Chemistry and Physics of Air Pollution. Wiley, New York.

Smirnov, V. I. (1987) Microstructure of clouds and deposits. Ann. Sci. Tech. (Meteor. Climatol.) 15, 3-193, Moscow (in Russian).

Subba-Ramu, M. C. and Muraleedharan, T. S. (1986) Formation of aerosols in irradiated atmospheric air. J. Aerosol Sci. 17, 745-752.

Sutugin, A. G. (1971) On the size dependence of the condensation coefficient. J. Statist. Phys. 1, 91-92.

Sutugin, A. G. and Grimberg, A. N. (1976) Quasi-two-dimensional description of the condensation in a free turbulent jet. $J$. Aerosol Sci. 7, 447-456.

Sutugin, A. G., Grimberg, A. M. and Puchkov, A. S. (1981) The multistate kinetics of the transition type nucleation in a free turbulent jet. J. Aerosol Sci. 12, 1025-1032.

Tohno, S. and Itoh, M. (1993) Production of highly concentrated nanophase Ag dispersoids without aggregation. J. Aerosol Sci. 24, 339-348.

Van Dingenen, R. and Raes, F. (1991) Determination of the condensation accomodation coefficient of sulphuric acid on water-sulphuric acid aerosols. Aerosol Sci. Technol. 15, 93-106.

Warren, D. R. and Seinfeld, J. H. (1984) Nucleation and growth of aerosol from a continuously reinforced vapor. Aerosol Sci. Technol. 3, 135-154.

Yalamov, Yu. I., Ivchenko, I. N. and Muradyan, S. M. (1981), Theory of evaporation od spherical droplets at arbitrary Knudsen's numbers. Dokl. Akad. Nauk SSSR 258, 1106-1111.

Ying, J. I. (1993) Structure and morphology of nanostructured oxides synthesyzed by thermal vaporization/magnetron sputtering and gas condensation. J. Aerosol Sci. 24, 315-338. 